\title{
DEVELOPING A CONSTRUCTION INTEGRATED MANAGEMENT SYSTEM
}

\author{
Yan-Chyuan Shiau, Ming-Teh Wang, Tsung-Pin Tsai, Wen-Chian Wang \\ Associate Professor \\ Department of Construction Engineering \\ Chung Hua University \\ Email:ycshiau@ms22.hinet.net
}

\begin{abstract}
Building market has been shortened for last few years and tremendously affected the construction profit in Taiwan. In order to survive in such abominable environment, people have put many efforts on construction methodology, materials, budget planning, and strategy alliance. However, to properly enhance the construction management is the key issue to raise company's competition. Therefore, an effective administrating tool is a must in construction life cycle such as estimating, bidding, procurement, scheduling, site managing, and valuation. This research has utilized Object Oriented concept, E/R techniques and Delphi environment to develop the Construction Integrated Manage System (CIMS). Through the help of this tool, user can effectively generate Bidding List, Purchasing Materials, Labor and Material Analyze Items and Specifications. Basic Database System can help us to modify resource prices to adjust budget, effectively control project cost and financial planning. Budget Control System will inform engineers when overspend occurs. Purchasing System can assist us to select the best subcontractors with most reasoning price. Valuation system can extract money request data from site records; therefore, prevent the over-calculating and short-calculating to achieve a better financial administration. With the help of CIMS, we can effectively control all details such as bidding, purchasing, contracting and evaluating, for each construction stage. This integrated system can practically control all information, maximize construction management efforts, raise company's strength, increase competition, and create profit for construction industry.
\end{abstract}

Keywords: Construction Management, Cost Control, Delphi, Object Oriented.

\section{Introduction}

With the rapid development of information technology, the traditional business behavior is deeply affected by the e-Commerce, and the management strategies of traditional industries are undergoing a fierce evolutional change. The features of the e-Commerce include:
A. Fast Speed - Real-time service, quick re- sponse and update.
B. Large Quantity - Simultaneity, automa- tion and diversity.
C. Lower Failure Rate - Digital data trans- mission reducing personal input errors.
D. Consecutive Service - 24-hour and 7-day service for the whole year.

With the aforementioned advantages, the services rendered by e-Commerce are better in quality than the services rendered by traditional providers. The traditional service providers are much weaker in competitive strength in comparison with e-Commerce companies as they are restricted by manpower. Thus, it is required to respond to the development of this tendency to enhance the productivity and competitive strength of the traditional building industry

To assist the industry in achieving the aforementioned goal, the "Integrated Information Management System for the Building Industry" is developed to integrate vertically the estimate, price assessment, bidding, price comparison, construction management, price calculation, payment request, and cash flow management. All the relevant information during the life cycle of a project I, and the calculation result of each pre-operation is stored in the system for future use to avoid any errors caused by repeated calculations and inputs.

\section{Structure and Function of the System}

The "Construction Integrated Management System" comprises the following modules:
A. Basic Database
B. Budget Module
C. Procurement Module
D. Price calculation Module
E. Settlement Management Module 
To integrate the sub-modules successfully, the study uses the object-oriented and network development tools to develop the modules. The function of each sub-module is described as follows:

\section{2-1 Basic Database}

Each building project is unique in its nature. Two buildings may have the same appearances, but the underlying bearing capacity and the conditions of neighboring houses are different from buildings to buildings. However, common characteristics exist among the components (column, wall, beam and formwork), materials (such as steel bar, concrete, ceramic tile), laborers (laborers in charge of reinforced steel, painting, plumbing) and documents (contract, construction instructions) used for a building.

Since such information is common for different projects, it is very useful to arrange it for future use so as to significantly educe the workload for data management and apply the cumulative experience for future projects.

The data contained in the database of the system include the basic resource data, work data, material analysis data, project instance data, subcontractor's data, labor data, project data, owner's data and database maintenance data (Fig. 1).

\subsubsection{Basic Resource Database}

The Basic Resource Database contains the data of manpower, machinery, materials, and other data concerning the resources to be used for the work.

\subsubsection{Project Items}

The project items are the data contained in bid documentations. In order to cope with complex information for a project, we need to use Object Oriented concept to mimic real world data by different objects. Based on the WBS [1], we make out the construction project items into three levels (Fig. 2): Large Items (15 items), Medium Items (341 items) and Small Items (3583 items). In this structure, Large Items are used for the high level items of the project (such as Reinforced Concrete Structure Engineering); Medium Items are used for the middle level items of the project (such as Systematic Formwork) and Small Items are used for the low level tasks of the project (such as DOKA column formwork).

\subsubsection{Material Analysis Data}

The cost of a project is acquired by analyzing the costs of material, labor and machinery. All the quantities and types of material, labor and machinery for are created in the system for the use of decision makers.

\subsubsection{Project Data}

In addition to the numbering, name, location and type of a project, the project data contains the data about the client number, contract number, commencement data, finish data, timeframe, contract price, contractor number, and designer name.

\subsubsection{Subcontractor's Data}

The Subcontractor's Data contains the data about (Fig. 3):

A. Basic Data (such as name, address, uniform number, capital and performance)

B. Business Items (Set upon the selection from the database)

C. Evaluation Data (including construction capability, reputation and financial status)

D. Unit price (Quoted by each subcontractor)

\subsubsection{Owner's Data}

The Owner's Data provides the data about the unit number, telephone number, responsible person, address, e-mail address, uniform number of the construction company concerned, project name, contact person, remark and other data related to the owner.

\subsubsection{Database Maintenance Data}

The cost structure differs significantly from projects to projects due to the difference in the location, timeframe, working environment, and nature of projects. To reflect the actual status, the user is allowed to set different construction conditions for different projects. The variables are adjustable according to the budget of each project and, in doing so, the user may control the progress of the construction. When the unit price of any resource changes, the user may adjust the cost and budget of the project concerned easily with the assistance of the internal calculation of the database. 


\section{2-2 Budget Module}

The Budget Module is used to calculate the construction cost based on drawings, rules and other documentations. The system uses individual works of the project concerned as a basis to estimate the quantities of labor, machinery and material required for each unit, which will further be used as a basis for the procurement and price calculation operations.

The Budget Module is designed based on an open structure to allow the user to define the smallest budget unit in accordance with awarding and procurement. The awarding can be conducted by combining the labor and material, depending on the nature and works involved. The Budget Module of the system provides the functions of budget preparation, budget adjustment and bid document preparation.

\subsubsection{Budget Preparation}

The system divides the budget of a single work into several categories to reflect the actual status. The divided budget includes the construction budget, awarding budget, execution budget and closing budget. The system creates different project budgets based on selected databases, work instances and reversed designs.

\subsubsection{Budget Adjustment}

In general, the budget may be affected by some factors appearing during the budget preparation phase and needs to be adjusted. The system allows the user to adjust the budget by one of the following approaches:

A. Adjust the total work price based on a fixed percentage.

B. Estimate and evaluate the possible change in price index in accordance with the time set in the Construction Progress Sheet for different resources.

C. Adjust the unit price of a resource that may be affected by external elements and cause the price to rise significantly. The overall change of the work can also be calculated in this step.

\subsubsection{Bid Document Printing}

The Bid Document Printing function of the Budget Module is used to print bid documents. All documents of a project, or the data of indi- vidual works, units, houses or buildings can be printed separately or integrally without problems.

\section{2-3 Procurement Module}

The procurement is the first step for a company to gain profits. The management may use the Procurement Module provided by the system to control dynamic market prices, conduct price negotiation, build advantageous niches and create the highest profits. The Procurement Module provides the functions of:

\subsubsection{Price Enquiry}

Before the awarding is conducted, it is required to analyze the instructions, quantities and rules of the project and print relevant data, so as to control the site conditions and other information before quotation is made and minimize the possible problems that may be encountered during the construction. The system is capable to integrate the resources required for the project and analyze relevant works and materials before sending the price enquiry sheet. It will also prepare construction instructions and unit price analysis sheets for subcontractors to arrange their resources. The system provides the following two price enquiry approaches:

A. Announcement via Network: The system will announce the awarding information via network, including the name, location, works, scale, rules and instructions of the project. The subcontractors may register, enquire and print on line.

B. Price Enquiry Sheet Printing Operation: The price enquiry sheet provides the information of awarding, project type, quantity, and unit price analysis and construction instructions. The printed price enquiry sheets will distributed to subcontractors so that they may arrange their resources accordingly (Fig. 4).

\subsubsection{Price Negotiation and Comparison}

The quotation of the subcontractors input in the system and the data imported by the subcontractors on line are integrated to make a price comparison sheet. After the identities of the subcontractors are confirmed, the price comparison sheet and the awarding budget will be considered carefully to form a basis for price negotiation. 
The system will integrate the data provided by subcontractors and calculate the total score of each subcontractor objectively based on different evaluation criteria and weights (including the construction capability, management capability, financial status, reputation and market advantages) [2].

\subsubsection{Contracting Documentations}

This function is used to investigate what projects and works a subcontractor has ever performed and the experience, financial status and management capability of the subcontractor.

\subsubsection{Material, Labor and Machinery Re- quirement Sheet}

This function is used to print the type, specifications and quantity of the material, labor and machinery required by a subcontractor to perform the work.

\section{2-4 Price calculation Module}

The Price calculation Module is used to transfer the data about the works and quantities from the site daily report system, and perform the price calculation for the current works and quantities of the site. The system will read the data of the contract quantity, procurement counts and cumulative payment request counts for cross comparison and analysis. When finding that the payment request counts exceed the procurement counts, the system will give an alert. The management may control the expected and actual construction status from the system and prevent the occurrence of possible problems as early as possible to minimize the risk of loss.

\section{System Development}

The system is developed through the following steps:

\subsection{Analysis of Existing Systems and Col- lection of Relevant Information}

The existing construction management systems available in the domestic market are investigated in the study to find out their shortcomings and the solutions to meet the demands of construction companies. The study also analyzes the historical records of different projects to investigate the difference in their nature and create an integrated system that is capable to handle multiple projects.

\subsection{Analyses of System Features and User Demand}

The process analysis technique is used to determine the functions and information flow processes required for the system. All unnecessary functions or processes are removed to simplify the maintenance of the system. The safety system is analyzed thoroughly by investigating individual potential problems and risks of safety, their affection and possible solutions.

Surveys are done to users about their demand for the "Integrated Information Management System for the Building Industry" to determine the requirements for each module, data type, printing format and other functions, in the hope to provide the best interface operation features for the industry.

\subsection{Creation of the Basic Data Framework}

The data processed by the system comes from the database. Therefore, it is crucial to create an effective integrated data framework. In addition to referring to the database structure of existing construction management systems, the study also takes the habits of users into consideration to create a basic data framework that are appropriately integrated for each module.

\subsection{Programming of Each Subsystem}

The study uses Delphi and BDE Administrator as the development tools and makes use of the network database writing technique to write subsystem programs. In addition to the single PC operation mode and the client/server design concept, the system integrates the Internet and multi-platform features. The standard Windows ${ }^{\mathrm{TM}}$ interface format is used to minimize the difficulty in operation.

\subsection{Test of System Functions}

In addition to confirming that the functions designed for each module can run as expected, the functions are tested to make sure that the system can meet the demand of the industry. The practical instances of the industry are collected for the test, and the comments of the users are analyzed for the modification of each subsystem. The integrated test is performed after all the functions of each subsystem have 
been tested and confirmed. The objective of the integrated test is to make sure that the data stream runs smoothly between different modules. The users confirm it after trial use that the system is capable to demonstrate the functions set for the industry and meet its demand.

\section{Database Structure}

\subsection{Classification of Works and Analysis of Materials}

The work data in the database is divided into three levels: Large Items (15 items), Medium Items (341 items) and Small Items (3583 items). Users may use the work data to create their own project data easily. The system also provides the material analysis sheet for each work, and all the labor, machinery and material fields in the material analysis sheet have their own resource numbering and quantity data. The users may change or update the data stated in the material analysis sheet from time to time and aggregate them to acquire the unit price of the work concerned.

\subsection{Engineering Resources}

The system contains 1100 resources and is capable to analyze the resources required for each work. The users may use the system to improve their finance management capability effectively, control the budget before commencing the procurement procedure, and prevent the cost from exceeding its allowed range. The database structure of the system is built with ER/Studio (Fig. 5).

\section{Conclusion}

The system is capable to integrate the tasks of a project, including planning, design, estimate, budget, procurement, documentation and price calculation. It is a very useful tool for the management of a construction company to control its projects effectively. The system provides the following functions:

\subsection{Basic Database}

The system divides the works to 17 large items, 341 medium items and 3583 small items, and integrates 1200 types of resources.

\subsection{Budget Module}

The system creates the project budget, awarding budget, execution budget and settlement budget based on the data in the database, individual projects and the latest plans. It also provides three approaches to adjust budget plans.

\subsection{Procurement Module}

The system provides a very useful technique for the selection of subcontractors and the determination of their weights, and allows users to announce the project to be awarded via Internet. The users may also use the system to print the quotation submitted by subcontractors for price comparison and negotiation, and select appropriate subcontractors in a fair and objective manner.

\subsection{Price Calculation Module}

The Price Calculation Module provides the works and quantities based on the data in the daily report system, and performs the price calculation for the current works and quantities of the site. The system will read the data of the contract quantity, procurement counts and cumulative payment request counts for cross comparison and analysis.

With the assistance of the system, construction companies may effectively control the information they need and integrate vertically the budget development, procurement and price calculation to carry out the construction management more efficiently. The system is capable to integrate the information scattered at different sites via Internet in a real-time manner to optimize the construction management of the construction company concerned, improve its competitive strength, enhance its organization, and bring it more profits.

\section{Acknowledgement}

I would like to take this opportunity to show my appreciation to National Science Council, Executive Yuan, for its support of the study. Project No.: NSC 90-2211-E-216- 015 。 


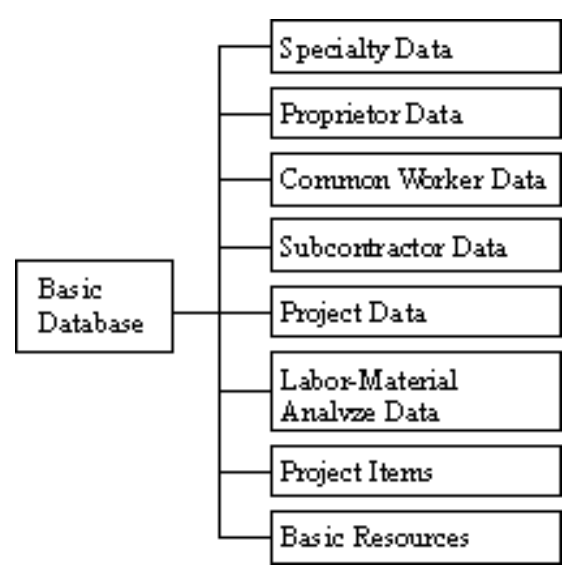

Fig. 1 Data Structure of the Basic Database

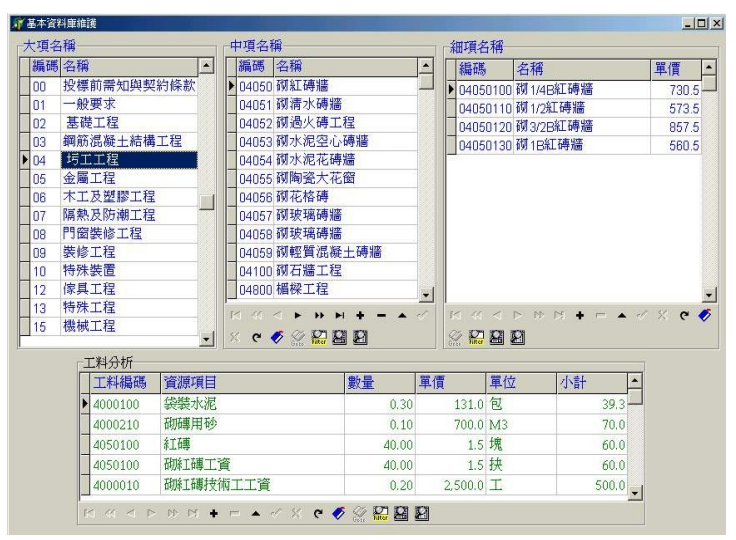

Fig. 2 Construction Project Dialog

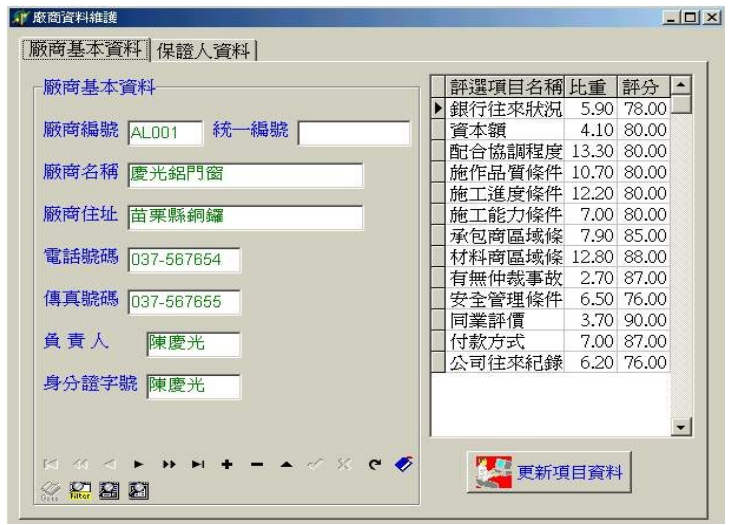

Fig. 3 Subcontractor Maintain Dialog

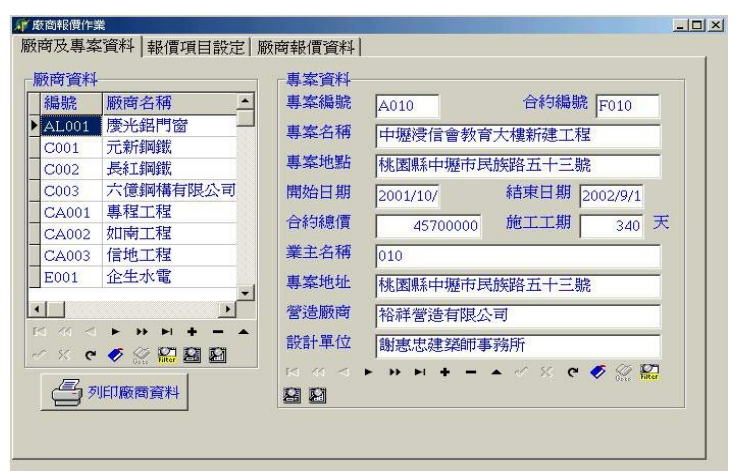

Fig. 4 Subcontractor's Quotation Dialog

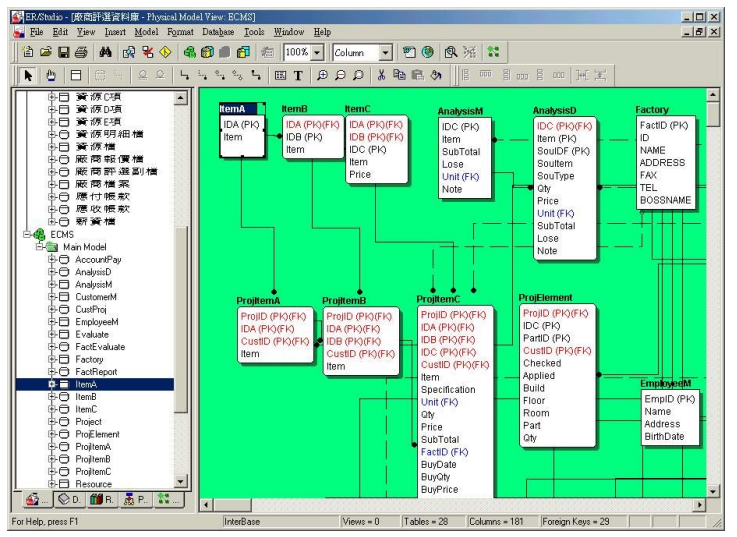

Fig. 5 Database Structure of the System

\section{REFERENCE}

1. Xing-Dao Zhang, "WBS, the First Step to Integrate Cost and Schedule", Construction Management, pp40 44.

2. Zong-Bin Cai, "Research on Procurement for Building Projects", A Master Dissertation, Department of Construction Engineering, Chung Hua University, Xin-Zhu, Taiwan, pp55 56, 2001.

3. Fouad M. Al-Sinan and Donn E. Hancher"Facility Project Delivery Selection Model,"Journal of Management in Engineering, ASCE, Vol.4, No.3, pp224-259, 1988.

4. Jian-Yu Chen and Zhong-Cheng Luo, "Contract Management for Government Project", Universal Law-108, pp29 30,1999.

5. Xian-Zhang Wu, "A General Discussion for Construction Contract Problem", Construction World, pp37 41,1999. 